Research Article

\title{
Simulation of Steady-State Temperature Rise of Electric Heating Field of Wireless Sensor Circuit Fault Current Trigger
}

\author{
Yinan Zhao $\mathbb{D}^{1,2}$ Jinwu Zhuang, ${ }^{1}$ Zhihao Ye, ${ }^{1}$ Zhiliang Qian, ${ }^{2}$ and Fang Peng ${ }^{2}$ \\ ${ }^{1}$ College of Electrical Engineering, Naval University of Engineering, Wuhan, 430033 Hubei Province, China \\ ${ }^{2}$ Wenzheng College of Soochow University, Suzhou, 215000 Jiangsu Province, China \\ Correspondence should be addressed to Yinan Zhao; wzj104@suda.edu.cn
}

Received 1 September 2021; Accepted 17 September 2021; Published 30 September 2021

Academic Editor: Guolong Shi

Copyright (c) 2021 Yinan Zhao et al. This is an open access article distributed under the Creative Commons Attribution License, which permits unrestricted use, distribution, and reproduction in any medium, provided the original work is properly cited.

\begin{abstract}
This article analyzes the structure of the wireless sensor circuit, considering the balance of power consumption, integration, area, noise, etc., and adopts a radio frequency wireless sensor circuit with a low-IF structure. Through the analysis and comparison of traditional analog current trigger and digital current trigger structure, the feed-forward current trigger structure is selected, which is composed of received signal strength indicator (RSSI) and variable gain amplifier (VGA), which achieves low power consumption, fast stabilization time, and wide dynamic range design. The received signal strength indicator adopts the form of approximate logarithmic amplifier, five-stage double feedback loop structure, and realizes lower power consumption. In order to prevent the load current trigger from entering the speed saturation zone, a gain unit structure in which the superimposed current trigger is connected to the NMOS tube as the load is proposed. The test results show that the circuit has a good power consumption performance $(1 \mathrm{~mW})$ and at the same time $56.8 \mathrm{~dB} / \mathrm{m}$ sensitivity. In this paper, through the analysis of the current trigger system and the analysis and comparison of the existing variable gain amplifiers, the variable gain amplifier structure composed of the folded wireless sensing unit and the index control unit is adopted. In order to reduce the power consumption of the circuit and increase the output swing, a structure in which the two-stage folding wireless sensor unit shares the controlled voltage-to-current part of the circuit is proposed. Aiming at the design requirements of the system, this article discussed in detail the architecture of the entire temperature measurement node and the design parameters of the chip and completed the overall architecture design of the chip. The simulation results of the steady-state temperature rise of the electric heating field show that the circuit has achieved an input dynamic adjustment range of more than $60 \mathrm{~dB}$, the maximum power consumption is $1 \mathrm{~mW}$, and the linearity error is less than $0.5 \mathrm{~dB}$. The designed automatic gain control circuit is implemented in SMIC 0.18 cape CMOS process. The simulation results of the steady-state temperature rise of the electric heating field show that the circuit has a $56 \mathrm{~dB}$ input dynamic adjustment range within a linear error of $1.25 \mathrm{~dB}$, and the time constant is $7.55 \mathrm{~ms}$, and power consumption is $2.84 \mathrm{~mW}$. Through the steady-state temperature rise simulation and test results of the electric heating field, the correctness of the design is verified and it meets the system requirements.
\end{abstract}

\section{Introduction}

With the rapid development of wireless communication technology, sensor technology, system-on-chip technology, etc., the wireless sensor network (WSN), called the peripheral nerve of the Internet of Things, has once again become a research hotspot at home and abroad and is widely used in military defense, industrial control, and environment surveillance and medical and health fields [1]. The radio frequency wireless sensor circuit is an important interface for wireless communication in the wireless sensor network, and its performance directly determines the quality of communication [2]. With different working environments and changes in distance, the signal power received by the antenna has a large dynamic range. In order to provide the optimal output amplitude for the baseband processing, the automatic gain control (current trigger) circuit is particularly important [3]. The research of this paper is based on the low-IF structure radio frequency wireless sensor circuit system, focusing on the design and chip realization of the 
automatic gain control circuit, and verifies the correctness of the design through the steady-state temperature rise simulation test of the electric heating field [4]. The wireless sensor network is a multihop self-organizing network, which is formed by a large number of cheap micro sensor nodes in the monitoring area through wireless communication [5]. The wireless sensor network connects the physical information of the objective world through a wireless network, which greatly expands people's ability to obtain information and provides people with the most direct and true information [6].

The wireless sensor network is mainly composed of a large number of sensor nodes. As the basic unit, the sensor nodes are the main task objects for collecting and transmitting information. They all have the characteristics of small size, low cost, ability to collect and process related data, and wireless communication functions [7]. The life span of the sensor node is determined by the battery that provides energy. The volume of the node itself is relatively small due to the requirements of the application environment, so the size of the battery is correspondingly limited, and the energy it has is also substantial [8]. And it is because many applications of sensor nodes require the use of unmanned conditions, so that the network can extend the working time as much as possible without replacing the battery, which has become another hot spot in the research of wireless sensor networks [9]. As the only energy source of the entire sensor node, the battery needs to support the subsequent detection and information processing of the node, but the operating voltage required by these parts is greater than the voltage value that the battery can provide. The battery voltage is boosted to the power supply voltage required by the subsequent circuit. In order to extend the battery life on the premise that the stored charge of the battery remains the same, we improve the conversion efficiency of the switching power supply, reduce its power consumption is the best way, and at the same time compress the area of the switching power supply integrated in the node to be smaller, so that the performance is optimized [10].

According to the performance indicators and functional requirements required by the system, this paper designs and analyzes the structure of the circuit system, studies the selection of peripheral circuits, and finally proposes the wireless sensor circuit hybrid electric heating field steady-state temperature rise modulation method. The chip system designed in this paper has a working frequency of $600 \mathrm{kHz}$, a stable operating temperature of $-40^{\circ} \mathrm{C}$ to $125^{\circ} \mathrm{C}$, an input voltage range of $3 \mathrm{~V}$ to $20 \mathrm{~V}$, an external inductance of $2.2 \mu \mathrm{H}$, and built-in undervoltage protection and current-limiting protection with overtemperature protection and soft start and other chip protection functions. The thesis then explained the working principle, circuit design, and electric heating field of important modules of the chip system including bandgap reference circuit, linear regulator, current limit detection circuit, peak current detection circuit, undervoltage latch circuit and over temperature protection circuit for simulation verification, and analysis of steady-state temperature rise. Based on the $0.35 \mu \mathrm{m}$ BCD standard process, the boost circuit chip system designed in this paper can be obtained after circuit design and steady-state temperature rise simulation analysis of the electric heating field under typical application conditions. The collision problem of wireless data transmission is a more troublesome problem in this system, because the sensor node in this system does not have a wireless receiver, so there is no synchronization method between the sensors. The output voltage ripple of the chip system can reach about $40 \mathrm{mV}$. The rate is $0.65 \% / \mathrm{V}$, and the actual conversion efficiency can reach more than $90 \%$. The chip meets the initial design indicators and functional requirements of the system. A new circuit is obtained through theoretical calculations, and then, through the ADS schematic diagram, layout, and the RF circuit calculated by cosimulation, the simulation parameters of the two RF circuits are compared, and finally the improved circuit is tested, and the network distance and diffraction experiments are passed. Finally, the Cadence software is used to simulate the steady-state temperature rise of the overall electric heating field under different input voltage and output load conditions.

\section{Related Work}

Power conversion circuits for self-powered wireless sensor nodes have long been a research hotspot at home and abroad, but there are very few power conversion products that can be used in actual power conversion. The reason is that high conversion efficiency under low input power has not been achieved. In recent years, with the continuous improvement of people's demand for quality of life, the research on wireless sensors has gradually shifted from military applications to people's daily life applications [11]. At the same time, with the rapid development of system-onchip (SOC) and very large-scale integrated circuit (VLSI), wireless sensor chips are developing towards miniaturization and low power consumption, wireless transmission, and multiple intelligence. With the rapid development of wireless sensor networks in recent years, the demand for highefficiency power converters has become more and more urgent, and the market has become larger and larger [12].

Bendary et al. [13] of the Virginia Institute of Technology in the United States and others have developed a wireless sensor that collects the vibrational mechanical energy of the human heart. The energy harvesting device can output $2.9 \mathrm{~mW}$ power under the excitation of $50 \mathrm{~Hz}$ vibration frequency, which can be used for structural health inspection. The self-powered technology based on vibrating mechanical energy has the advantages of small size, process compatibility with traditional semiconductor processes, and a wide range of application environments. However, the disadvantage is still that the power is small, and the integration of the vibrating mechanical energy collection device is not high, and the assembly is difficult. Although the technology of large-scale power generation devices is mature, it is not suitable for wireless sensor nodes. Therefore, small-scale wind power generation technology with small size and low wind speed requirements has been developed. Gomati et al. [14] used piezoelectric technology to create a small wind turbine with a power of $50 \mathrm{~mW}$, with an efficiency of $11 \%$. Wu 
et al. [15] of the University of Brescia and others designed a wireless sensor node for monitoring air temperature and wind speed based on wind power generation. The wind power generation device can output $45 \mathrm{~mW}$ at a wind speed of $9 \mathrm{~m} / \mathrm{s}$. The space is full of electromagnetic waves, and electromagnetic energy can be converted into electrical energy by using the principle of electromagnetic induction. However, the electromagnetic wave energy density in space is limited, and the power of this self-powered method is too low, so it is generally used in places with high electromagnetic field strength such as substations. Zaghari et al. [16] of the University of South Preston in the United Kingdom developed a wireless sensor node that collects electromagnetic vibration energy and provides an output power of $58 \mu \mathrm{W}$.

As an important part of the wireless sensor network, the wireless sensor chip has always been a research hotspot in academia. Professor Ji et al. [17] developed an ultralow power temperature sensor module based on food monitoring that can be integrated on RFID tags. The chip biases the MOS tube of the sensor in the subthreshold region, which makes the power supply voltage of the sensor part as low as $0.5 \mathrm{~V}$, thereby greatly reducing the power consumption of the module. At the same time, the sensor uses an inverter chain temperature measurement method; this method has a lower accuracy than the traditional BJT-based temperature measurement method, but the circuit is simple and the power consumption is lower. The experiment measured that the power consumption of the chip is only $119 \mathrm{nW}$ at a sampling rate of 333 , and the temperature measurement accuracy can reach $+1 /$ $0.8^{\circ} \mathrm{C}$ (temperature measurement range -10 to $130^{\circ} \mathrm{C}$ ). However, the abovementioned circuit only stays in the module stage and only realizes the temperature collection and does not realize a complete sensor node. Lowvoltage domain and subthreshold circuit design can reduce the power consumption of digital circuits more significantly. Digital circuits can work in a very low-voltage domain at the expense of speed. At the same time, lowvoltage design also reduces the power consumption of digital circuits to a certain extent. The team of Professor Mazumdar [18] of the University of Michigan in the United States used the TSMC0.18 process to develop an intraocular pressure sensor with a volume of less than 1 , which is mainly used to help glaucoma patients monitor intraocular pressure in real time and prevent unnecessary blindness caused by abnormal intraocular pressure. The whole sensor contains two integrated chips: top chip and bottom chip. Top chips mainly include solar devices and wireless transceivers, while bottom chips mainly include capacitance-to-digital converters, microprocessors, and SRAM. In order to reduce the power consumption of the microprocessor and SRAM, VDD is reduced to $0.45 \mathrm{~V}$, which makes its power consumption only $90 \mathrm{nW}$. The entire sensor module is implanted into the human eye, and the sensor uses the light passing through the human cornea to obtain energy and transmits the intraocular pressure readings to the external receiving terminal through wireless communication [19-22].

\section{Construction of the Steady-State Temperature Rise Model of the Electric Heating Field of the Wireless Sensor Circuit Fault Current Trigger}

3.1. Hierarchical Distribution of Wireless Sensor Modules. The wireless sensor node is mainly composed of four parts: sensor module, processor module, wireless communication module, and energy supply module. Figure 1 shows the hierarchical distribution of wireless sensor circuit modules. Multiple wireless communication modules constitute a wireless communication system. After the data from the application interface is modulated and upconverted by the steady-state temperature rise of the electric heating field of the transmitter, it is radiated through the antenna and propagated in the communication channel. After the wireless sensor circuit receives the relevant signal, it undergoes downconversion and demodulation to restore the data to the original format and then transmits it to the application interface.

In the wireless communication module, the radio frequency transceiver plays a vital role, and its performance directly determines the quality of the communication. A wireless sensor network is a wireless network composed of stationary or moving sensors in a self-organizing and multihop manner. In the network coverage area, it is for the purpose of sensing, collecting, processing and transmitting the information of the sensing object, and sending it to users.

$$
X[t]=\{t \in R \mid x(i), \quad i=1, \cdots, n\}
$$

The wireless sensor network integrates the three disciplines of computer, communication, and information perception. From the perspective of computer science, it is a typical self-organizing multihop network; from the perspective of communication methods, it uses wireless communication methods, which can save a lot of data lines; from the perspective of information perception, its deployment purpose is to obtain information, and different sensor forms are required for different application scenarios.

$$
\nabla^{2} \frac{\alpha-1}{a^{2}+1} \times \frac{\partial^{2} \alpha}{\partial t^{2}}+\frac{\rho}{\varepsilon}=0
$$

The basic function of wireless sensor network is to collect, transmit, process and store various information in the physical world, and then submit it to users who are interested in the information. In order to realize this function, the wireless sensor node includes the following modules: sensor module, processor module, wireless communication module, and energy supply module.

$$
y(n+1)=A \times X(n+1)-t \times x(n)
$$

Compared with traditional wireless networks (such as WLAN), wireless sensor networks have very different design goals. Traditional wireless networks mainly improve bandwidth utilization in highly mobile environments as much 


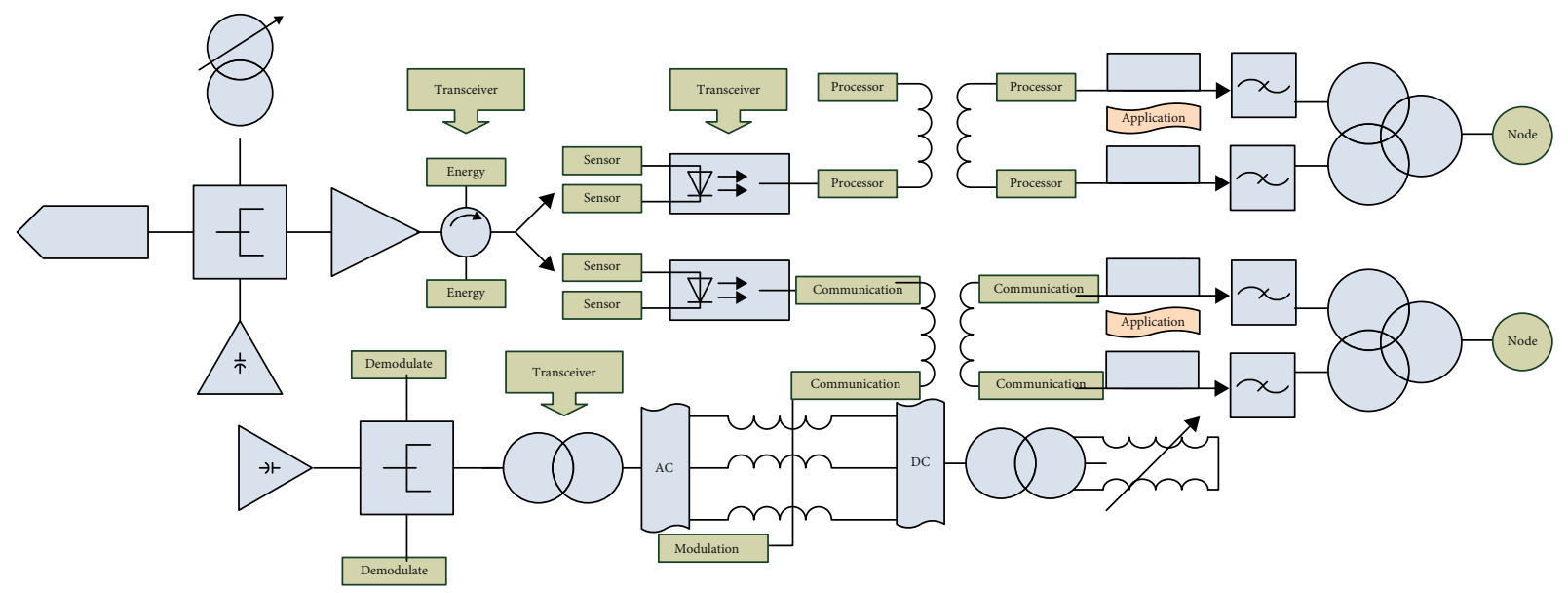

FIgURE 1: Hierarchical distribution of wireless sensor circuit modules.

as possible, while ensuring a certain quality of service.

$$
h(x)=\left\{\begin{array}{l}
\varepsilon(i, n) \times x(t), 0<t<1, \\
\delta(i, n) \times x(t), t>1, \\
y(n) \times x(t), t<0
\end{array}\right.
$$

In the wireless sensor network, most of the sensor nodes are stationary, and only a few nodes need to be moved. Its core problem is to design an effective strategy to extend the life cycle of the network as much as possible. Sensor networks also have technical requirements that are significantly different from traditional wireless networks. The former is data-centric, and the latter is data-transmitting.

$$
f(x+1)-f(x)=\partial \frac{y(x, n)}{\|x(n)\|^{2}} \times x(n) .
$$

It mainly includes distributed sensor nodes, aggregation nodes, task management nodes, the Internet, and satellites. Sensor nodes are usually randomly distributed in the monitoring area. Each node can collect information and route the information to the sink node through a multihop infrastructure-free structure.

$$
\overline{x^{2}}(t, n)-|x(t, n)|^{2}-2|\overline{x(t, n)}| \times \cos \alpha=0
$$

Then, the sink node communicates with the task management node through the Internet and satellites. The user configures and manages the wireless sensor network through the task management node, releases monitoring tasks, and collects monitoring data.

\subsection{Topological Structure of Circuit Steady-State} Temperature Rise. The so-called steady-state temperature rise modulation method of the electric heating field is to control the switching state of the power switch tube through a certain logic circuit to obtain the required output voltage and duty cycle and make the output voltage not change with external factors such as load or input conditions.

$$
\frac{\partial^{2} \alpha}{\partial x^{2}}+\frac{\partial^{2} \alpha}{\partial y^{2}}+\frac{\partial^{2} \alpha}{\partial z^{2}}-f(x)=0
$$

There are mainly four modulation methods for the steady-state temperature rise of the electric heating field of the converter: PWM electric heating field steady-state temperature rise modulation method, PFM electric heating field steady-state temperature rise modulation method, PSM electric heating field steady-state temperature rise modulation method, and hybrid electric heating field steady-state temperature rise modulation method.

$$
\left\{\begin{array}{l}
\frac{\alpha(x)-\alpha(x-1)}{\sum a(t) \times w(x)}=1, \\
\frac{\beta(x)-\beta(x-1)}{\sum b(t) \times w(x)}=1 .
\end{array}\right.
$$

The steady-state temperature rise modulation method of the PWM electric heating field means that when the load condition or the input voltage changes, the switching frequency is not changed, but the loop is adjusted by changing the duty cycle of the inductor charging and discharging time, so that the output voltage is maintained stable and unchanging. Figure 2 shows the fan diagram of the load modulation ratio of different circuit temperature rise topology modules. PWM has better conversion efficiency and linearity in a wide load range, especially under heavy load conditions; the response to load changes is faster, because the switching frequency remains the same. Compared with other electric heating, the field steady-state temperature rise modulation method is easier to filter out noise, which is a good choice for noise-sensitive systems. However, the efficiency is low under light load conditions, the static power consumption is relatively large, and due to the limitation of the minimum on-time of the switch tube, the adjustment range of the output voltage will be narrowed.

The reference voltage Vref is used as the positive input voltage of the error amplifier, and the feedback voltage 

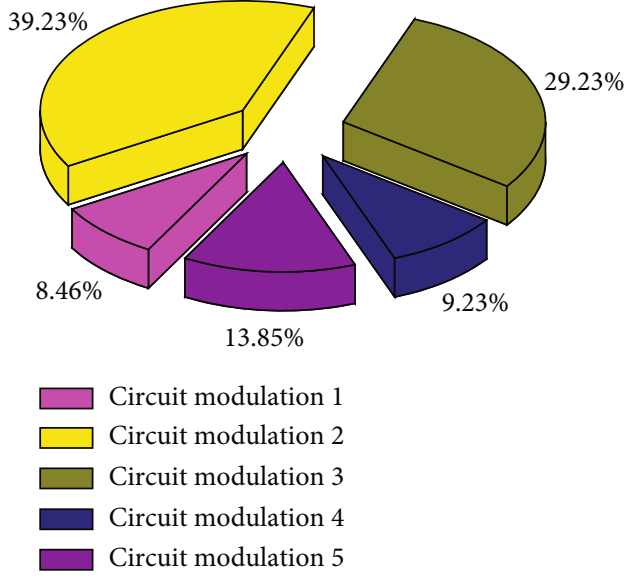

FIgURE 2: Fan diagram of load modulation ratio of different circuit temperature rise topology modules.

VFB (that is, the divided voltage of the output voltage) is amplified by the error amplifier EA to obtain the VCOMP signal. The signal is compared by the PWM comparator, and a PWM output result is obtained to control the switching state of the power tube. Assuming that the output voltage VOUT increases, the obtained feedback voltage VFB increases, the VCOMP signal output by the error amplifier decreases, and the output pulse width obtained by comparing with the constant frequency sawtooth wave is transformed accordingly, so that the charging time duty ratio of the power tube decreases to adjust the output voltage to a stable state. The difference between the feedback voltage obtained by dividing the output voltage and the reference voltage Vref is used for error amplification to obtain an error amplification signal, which changes the corresponding clock frequency through an oscillator. The entire temperature measurement node is a temperature measurement system with low power consumption wireless sensor temperature measurement chip as the core, self-energy module, DCDC, timer, and other off-chip modules as auxiliary temperature measurement system. Under normal circumstances, PFM has two ways: one is to keep Ton unchanged and change Toff, that is, to change the control signal by changing the length of the low potential while keeping the frequency signal at a constant high potential. There is another way to keep Toff unchanged and change Ton, that is, to change the duty cycle of the control signal by changing the duration of the high potential while maintaining the low potential of the frequency signal for the same time.

Figure 3 shows a schematic diagram of the circuit's steady-state temperature rise topology. When Vctl is high to turn on the power switch NM1, the rectifier current trigger $\mathrm{D}$ is reversely cut off, the voltage across the inductor $\mathrm{L}$ is VIN, and the input voltage VIN charges the energy storage element inductor $\mathrm{L}$. As time increases, the inductor $\mathrm{L}$ increases proportionally; when the Vctl signal changes from a high level to a low level, the power switch NM1 is turned off, the rectifier current trigger $\mathrm{D}$ is turned on, the voltage across the inductor L is VOUT-VIN, and the voltage direction is reversed. The voltage sources collectively charge the capacitor $\mathrm{C}$ through the rectifier current trigger $\mathrm{D}$. The inductor current decreases linearly with time, and the charge on the capacitor gradually accumulates to increase the output voltage VOUT. When the power switch NM1 is turned on, the inductor current increases proportionally with the increase of time; when the rectifier current trigger $\mathrm{D}$ is turned on, the inductor current decreases proportionally with the decrease of time. During the time when the power switch NM1 is turned off, the current of the inductor L drops to 0 , and the inductor $L$ keeps the stored energy at 0 during the remaining time when the power switch is turned off. This kind of switching power supply is called working intermittently with the inductor current. In the same way, if the minimum value of the inductor current drop is greater than 0 during the entire power switch NM1 turn-off period, the switching power supply is said to work in the inductor current continuous mode (CCM).

3.3. Optimization of Circuit Node Layout. The main task of the sensor node is to monitor and collect environmental information. The relay node mainly acts as a bridge to connect the sensor node and the routing node in order to expand the transmission distance. The main task of the control center is the establishment of the network and data forwarding. The node hardware in the three networks is almost the same, and the hardware can be distinguished through software. Generally, sensor nodes should have the following functions, that is, collecting information, processing information, and transmitting information. According to this first function, the sensor node should have the ability to collect such as temperature and light intensity, so the node hardware should have temperature and light sensors. In the wake-up mode, the energy supply module provides energy to the sensor node to make the sensor node work normally, and once the work is completed, the sensor enters the sleep mode. In the sleep mode, the power of most modules of the node is turned off, and the energy obtained by the self-power module is stored in the super capacitor until the next time the sensor is awakened and enters the wake-up mode. A large number of distributed wireless sensor nodes are an important part of the wireless sensor network, and they are the eyes of the wireless sensor network. Table 1 shows the hierarchical distribution of information transmitted by circuit nodes.

Each wireless sensor node is a small system, including sensors, microcontrollers, data storage, wireless transceiver modules, and power supply modules. The sensor node needs to upload the temperature information of the temperature measurement point every minute, so the entire sensor node needs to enter the wake-up mode once every minute, and then return to the sleep mode after uploading the temperature information to save power consumption. After the upload is complete, the digital control module of the sensor node will generate an "upload complete" signal to turn off the power switch again, so that the sensor node will return to sleep mode. Figure 4 shows the schematic diagram of the wireless sensor circuit node layout.

Different from traditional wireless sensor nodes, selfpowered wireless sensor nodes have energy harvesting 


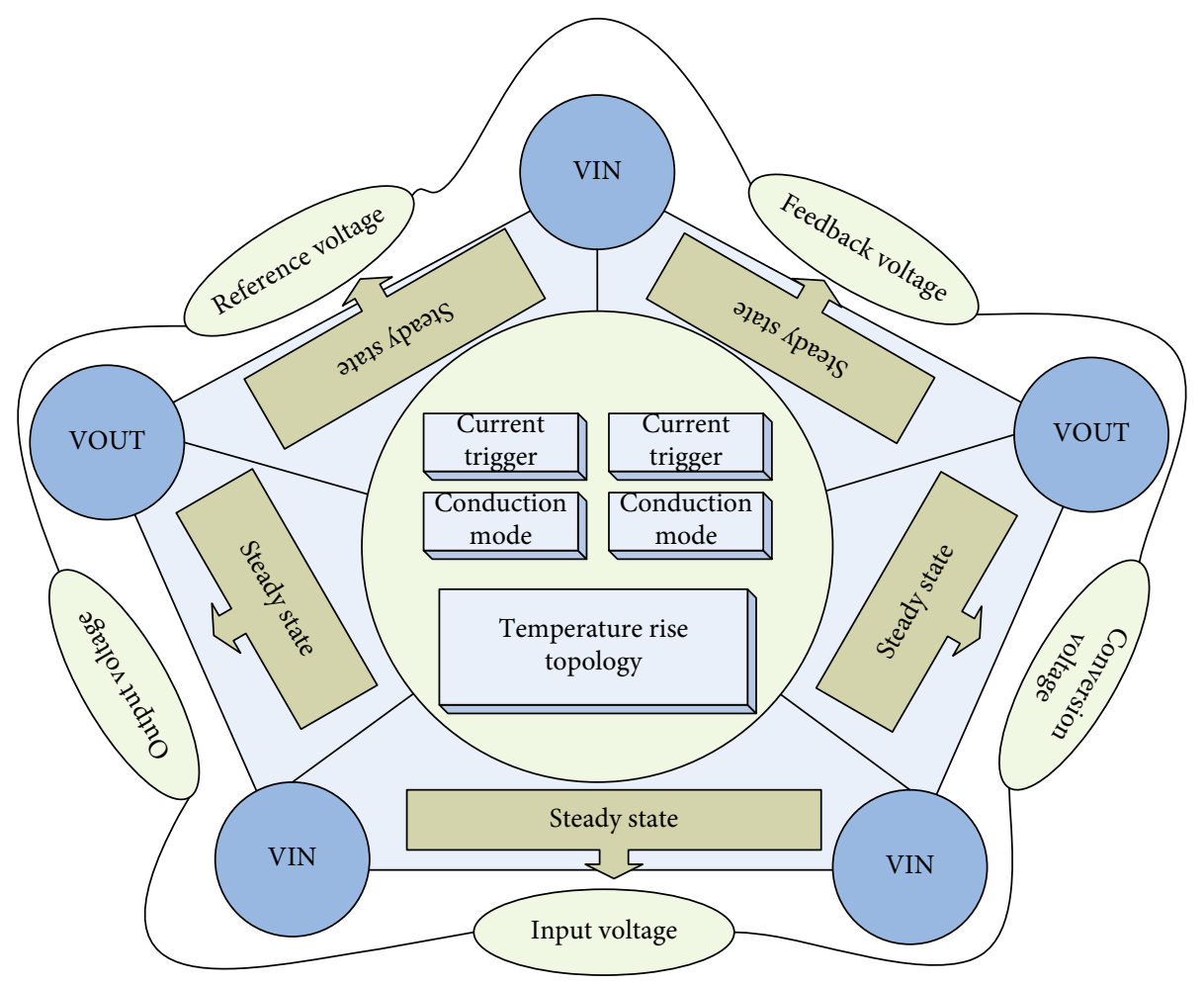

Figure 3: Schematic diagram of the circuit's steady-state temperature rise topology.

TABLE 1: Hierarchical distribution of transmission information of circuit nodes.

\begin{tabular}{lcccc}
\hline $\begin{array}{l}\text { Circuit } \\
\text { node index }\end{array}$ & $\begin{array}{c}\text { Temperature } \\
\text { range/ }{ }^{\circ} \mathrm{C}\end{array}$ & $\begin{array}{c}\text { Signal } \\
\text { delay/ms }\end{array}$ & $\begin{array}{c}\text { Dielectric } \\
\text { constant/(F/ } \\
\mathrm{m})\end{array}$ & $\begin{array}{c}\text { Poisson's } \\
\text { ratio }\end{array}$ \\
\hline 1 & $0-80$ & 21.5 & 3.5 & 0.31 \\
2 & $-10-80$ & 22.1 & 1.9 & 0.35 \\
3 & $20-100$ & 35.1 & 11.9 & 0.27 \\
4 & $-20-120$ & 31.7 & 2.1 & 0.29 \\
\hline
\end{tabular}

modules to obtain thermal energy, vibration mechanical energy, wind energy, light energy, and electromagnetic energy from the environment and achieve energy selfsufficiency. The energy harvesting device converts the acquired environmental energy into electricity in the form of energy, which is converted to other modules for use through the power conversion module, and the remaining power is stored in the energy storage device. The AC-DC circuit is composed of a voltage conversion loop and a feedback control loop, supplemented by an input and output protection circuit. The voltage conversion loop controls the output voltage through the transformer coil turns ratio and the switch duty cycle, and the feedback control loop compares the sampled voltage with the reference voltage to control the switch duty cycle. The voltage conversion loop and the feedback control loop form a loop to jointly control the output voltage to ensure the stability of the output. As the working speed of the circuit becomes faster and faster, the signal transmission speed becomes higher and higher. The PCB wire is not only a device interconnection tool. At this time, it must be ensured that it can accurately transmit the signal to the input end of the device. The whole temperature measurement node is a temperature measurement system with low power consumption wireless sensor temperature measurement chip as the core, self-energy module, DC-DC, timer, and other off-chip modules as auxiliary temperature measurement system. The temperature measurement chip completes the temperature collection, processing, and wireless transmission during the "enable" phase.

\section{Application and Analysis of the Steady-State Temperature Rise Model of the Electric Heating Field of the Wireless Sensor Circuit Fault Current Trigger}

4.1. Circuit Failure System Design. The wireless sensor node is divided into multiple modules according to the circuit function. According to the signal flow, the temperature sensor first converts the temperature of the collection point into an analog electrical signal, which is amplified by an amplifier and connected to an analog-to-digital converter (ADC). The analog signal is digitized by the ADC. After the digital controller receives the temperature digital code, it integrates the temperature information into a data packet that conforms to the communication protocol and transmits it to the wireless transmitter (TX) through the antenna. Figure 5 shows the oscillation frequency curve of the wireless sensor circuit. The PLL in TX receives the signal from the external crystal oscillator and generates a reference frequency. It can not 


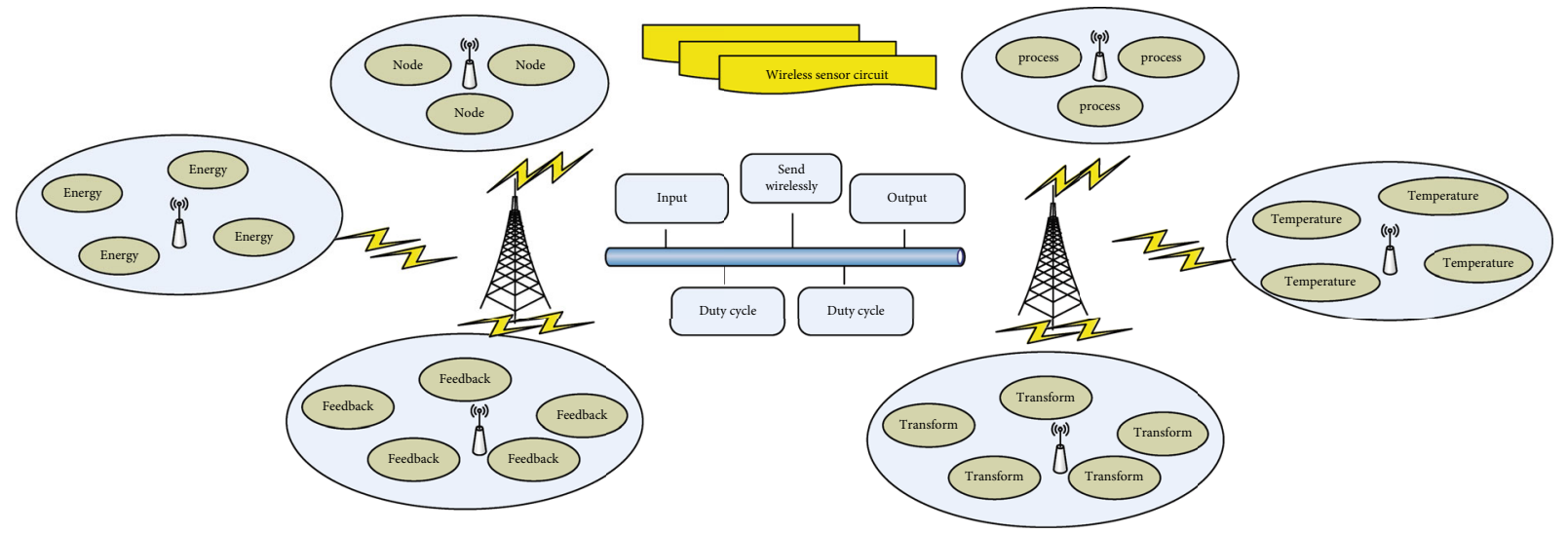

FIGURE 4: Schematic diagram of wireless sensor circuit node layout.

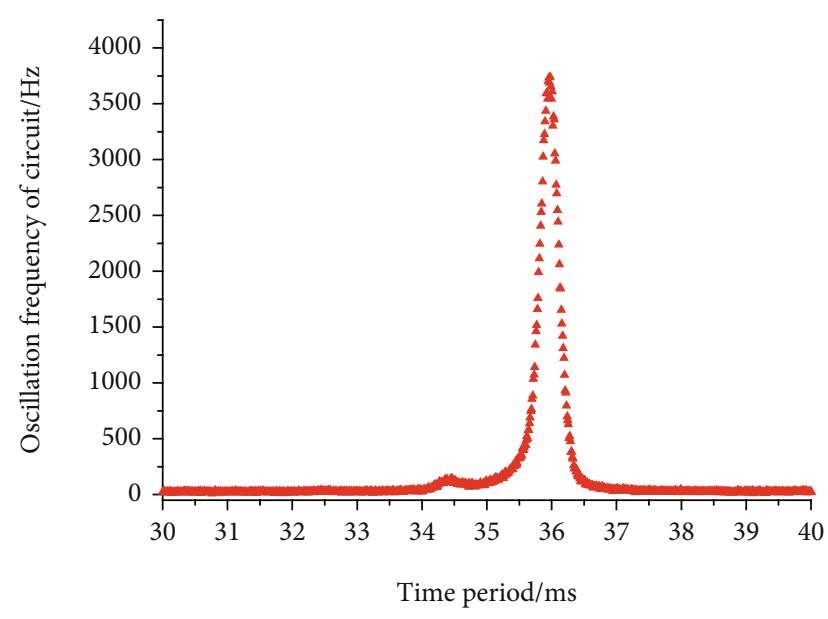

Figure 5: Oscillation frequency curve of wireless sensor circuit.

only be used as the carrier frequency when TX itself is transmitting, but it can also generate some clock signals through frequency division for ADC and digital controller. When I $=400 \mathrm{nA}$, the clock cycle is $11.36 \mathrm{~ms}$, and the clock frequency is $88.0 \mathrm{kHz}$; when $I=300 \mathrm{nA}$, the clock cycle is $13.32 \mathrm{~ms}$, and the clock frequency is $75.1 \mathrm{kHz}$; when $I=$ $200 \mathrm{nA}$, the clock cycle is $16.76 \mathrm{~ms}$, and the frequency of the clock is $59.7 \mathrm{kHz}$. As the load decreases, the oscillation frequency gradually decreases, thereby reducing the switching frequency of the system and reducing the system power consumption.

The input of the analog-to-digital converter is two sinusoidal signals with a common mode of $1 \mathrm{~V}$ with opposite phases and an amplitude of $0.7 \mathrm{~V}$, so that the differential input is a sinusoidal signal with $-1.4 \mathrm{~V} \sim 1.4 \mathrm{~V}$, the reference voltage is $1.4 \mathrm{~V}$, and Clks is a pulse width of $500 \mathrm{~ns}$, periodic square wave with a period of $2.5 \mathrm{~ms}$. At the same time, for the convenience of testing, a 10-bit ideal DAC is connected after the ADC. In the simulation of steady-state temperature rise of the electric heating field, the power supply voltage is $0 \mathrm{~V}$ at time 0 and reaches $2 \mathrm{~V}$ at $0.25 \mathrm{~ms}$. The design principle of the start-up circuit is that during the power-on process of the circuit, the start-up circuit works to make the bias circuit out of the degeneracy point. After the bias circuit works normally, the start-up circuit is closed and cannot affect the operation of other circuits. In the steady-state temperature rise simulation results of the electric heating field, it can be seen that the output of the temperature sensor is stable after about $3.5 \mathrm{~ms}$ after the power is turned on. This time is very sufficient for the entire system, because the stabilization time of the PLL is about $180 \mathrm{~ms}$ or so. A voltage (bandgap reference) that does not change with temperature is generated during the design of the temperature sensor. Under the XFAB $0.35 \mu \mathrm{m}$ process used in this design, the bandgap reference is approximately $1.2 \mathrm{~V}$.

In the circuit design, it is likely that other values of voltages that do not change with temperature are also needed. For example, the $\mathrm{ADC}$ in this system requires a $1.4 \mathrm{~V}$ reference voltage, so a reference voltage generation module is needed to generate these voltages using a bandgap reference. The sampling rate represents the number of data points that the analog-to-digital converter can output per unit time. The sampling rate of the analog-to-digital converter in this article is $400 \mathrm{~ms}$, which means that the analog-to-digital converter in this article can output $400 \mathrm{k}$ data points per second at the fastest. Figure 6 shows the distribution of scatter points of the temperature rise resolution of the electric heating field of the circuit. The average power consumption in the above table represents the average power consumption of the analog-to-digital converter at a sampling rate of $400 \mathrm{~ms}$. The ENOB of the analog-to-digital converter in this article reaches 9.75 bits, which means that the temperature measurement resolution can reach $0.163^{\circ} \mathrm{C}$ within the temperature measurement range $\left(-20 \sim 120^{\circ} \mathrm{C}\right)$.

\subsection{Steady-State Temperature Rise Simulation of Electric} Heating Field in Wireless Sensor Network. Here, we use Agilent's ADS (Advanced Design System) software to simulate the steady-state temperature rise of the electric heating field at the schematic level and layout level of the circuit. ADS is a steady-state temperature rise simulation software for microwave circuits and communication systems launched by Agilent. It is the most widely used software in the industry today. It is used in communication systems and microwave radio frequency circuits. In the schematic-level electric heating field steady-state temperature rise simulation, it can 


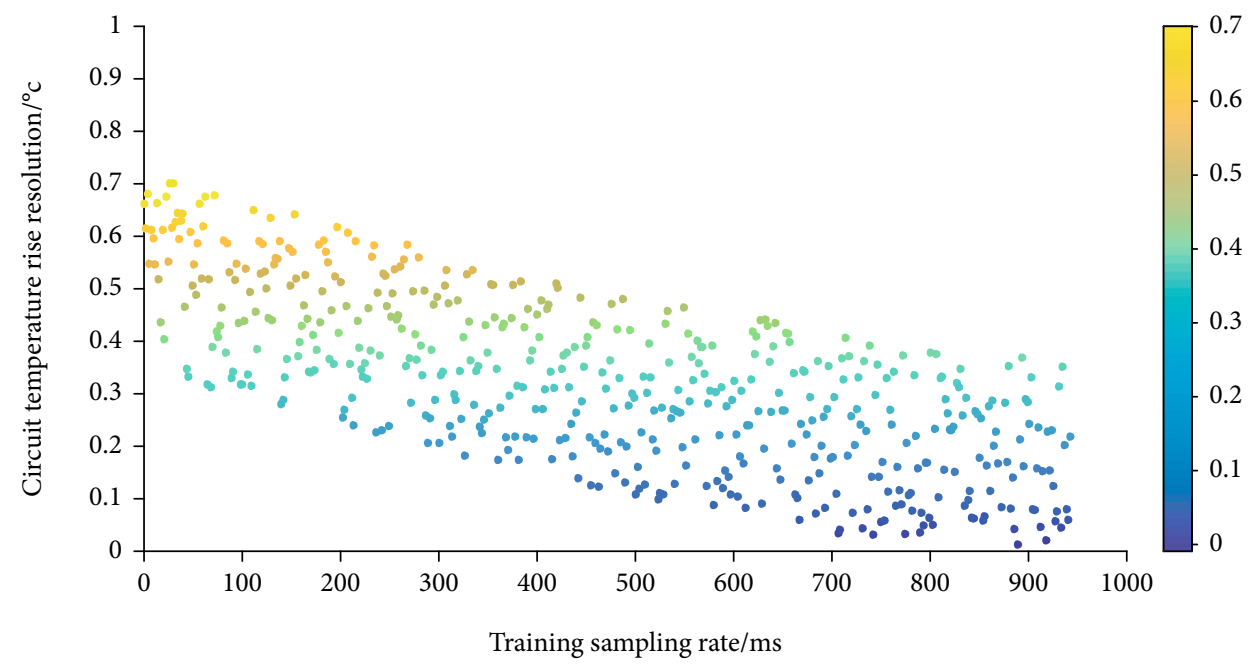

FIgURE 6: Scattered point distribution of the temperature rise resolution of the electric heating field of the circuit.

realize the steady-state temperature rise simulation of the electric heating field in the time domain and frequency domain, linear and nonlinear, and can analyze the design results and optimize the circuit. It is an excellent microwave $\mathrm{RF}$ design tools. And it can be directly interconnected with EDA software such as Cadence, and the produced PCB file can be directly imported into ADS to simulate the steadystate temperature rise of the electric heating field at the board level, which is very convenient for application. In the case of high frequency, the copper wire that transmits the signal on the PCB can be regarded as a combination of series equivalent resistance, inductance, and parallel capacitance. Knowing from the signal integrity knowledge, when the transmission line impedance does not match, problems such as reflection will occur, reflection question will reduce the transmission performance and affect the transmission distance, and effective circuit matching can make the signal energy flow from the power source into the load effectively. Figure 7 shows the trend of the loop gain of the wireless sensor circuit with the load. When the load current $I=1 \mathrm{~mA}$, the loop gain is $41 \mathrm{~dB}$ and the phase margin is 95 ; when the load current $I=100 \mu \mathrm{A}$, the loop gain is $61.6 \mathrm{~dB}$ and the phase margin is 56 . From the simulation results of the steady-state temperature rise of the electric heating field, it can be obtained that the LDO circuit can remain stable within the required load variation range.

The temperature compensation and overtemperature protection circuit designed in this article consists of PMOS tubes MP1 MP7, NMOS tubes MN1 MN5, resistors R1 R6, two capacitors, two transistors, two inverters, and one Schmitt trigger. The operational amplifier is composed of, among them, MP1 and MP2, MP3, MP4 and MP5, MP6 and MP7, MN4 and MN5 are all current mirror connections, and the operational amplifier OP, NMOS tube NM3, and resistor R3 form a negative feedback loop. These ports need to be set to the internal port mode, that is, in the layout electric heating field steady-state temperature rise simulation, it is regarded as the characteristic of the entire circuit board for the electric heating field steady-state tem- perature rise simulation, and the two ports of the differential line need to be set as differential ports. The mode corresponds to the actual circuit, and the antenna end is set to single-port mode. From the PVT simulation results, the output of the temperature sensor is slightly different under different process angles, and the DC deviation can be eliminated by single-point calibration. But under different process angles, the output linearity is better. From the perspective of comprehensive system requirements, the simulation results of the above circuit are acceptable. Two of the differential ports are set to $60 \mathrm{ohms}$ for the conjugate matching of the ports to achieve the maximum power reception at the antenna end, and the antenna port is set to $50 \mathrm{ohms}$ to meet the impedance matching of the antenna. Table 2 shows the port amplitude frequency characteristics of the wireless sensor circuit. After the above settings are completed, we select the RF simulation mode and set the scan frequency to $300-500 \mathrm{~Hz}$.

From the simulation results of the steady-state temperature rise of the electric heating field, it can be seen that when the load current jumps from $100 \mu \mathrm{A}$ to $1 \mathrm{~mA}$, the undershoot voltage is $122 \mathrm{mV}$ and recovers to $5 \mathrm{~V}$ after $2.7 \mathrm{~ms}$; when the load current jumps from $1 \mathrm{~mA}$ to $100 \mu \mathrm{A}$, the overshoot voltage is $148 \mathrm{mV}$, and the recovery time is $2.4 \mathrm{~ms}$. We can see that the $\mathrm{VH}$ voltage starts to rise from $5 \mathrm{~V}$ and needs to rise to be greater than the upper threshold voltage before the undervoltage latch circuit will output a high level. Similarly, when $\mathrm{VH}$ falls from $6 \mathrm{~V}$, it needs to fall to less than the lower threshold voltage. The voltage latch circuit outputs a low level. The upper threshold voltage $\mathrm{VU}=5.52 \mathrm{~V}$, the lower threshold voltage $\mathrm{VL}=5.31 \mathrm{~V}$ obtained by the steady-state temperature rise simulation of the electric heating field, and the hysteresis is $210 \mathrm{mV}$, which meets the design requirements.

4.3. Example Application and Analysis. This design uses the $\mathrm{XFAB} 0.35 \mu \mathrm{m}$ process, and the process manufacturer provides three design process angles for this process: tm (typical mode), wp (worst power), and ws (worst speed). The digital 


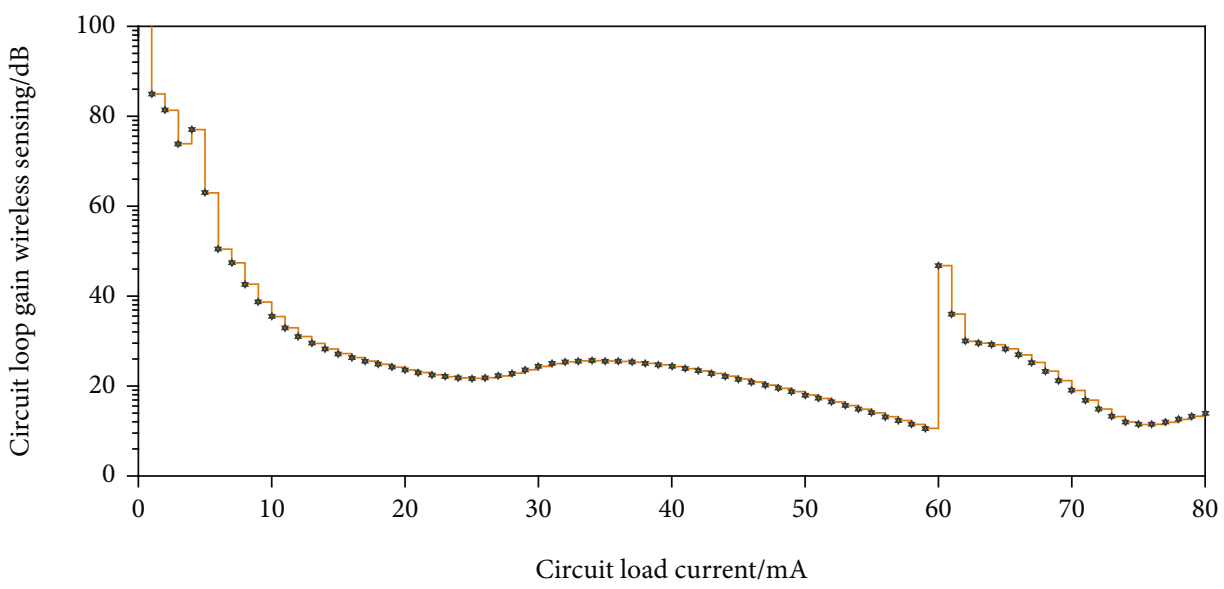

Figure 7: The loop gain of the wireless sensor circuit varies with the load.

TABLE 2: Amplitude frequency characteristics of wireless sensor circuit ports.

\begin{tabular}{lccc}
\hline $\begin{array}{l}\text { Circuit port } \\
\text { number }\end{array}$ & $\begin{array}{c}\text { Signal } \\
\text { identification }\end{array}$ & $\begin{array}{c}\text { Signal } \\
\text { amplitude/mV }\end{array}$ & $\begin{array}{c}\text { Signal frequency } \\
\text { band/Hz }\end{array}$ \\
\hline 1 & ECG & $0.1-5$ & $0.5-120$ \\
2 & EEG & $0.1-1$ & $0.1-100$ \\
3 & EMG & $1-5$ & $20-1000$ \\
4 & EAG & $0.1-2$ & $0.2-100$ \\
5 & EPG & $0.5-5$ & $1-1000$ \\
\hline
\end{tabular}

controller enters a waiting time of about 100 clock cycles, that is, $60 \mathrm{~ms}$, waiting for the initialization of circuits such as temperature sensors, amplifiers, and analog-to-digital converters. When the input voltage of the circuit is $250 \mathrm{mV}, 900 \mathrm{MHz}$, and the load is $20 \mathrm{M} \Omega$, the output voltage of the voltage doubler rectification can be obtained, and the final output can reach about $890 \mathrm{mV}$, which can reach the turn-on voltage of the wake-up circuit, and can provide power for the subsequent stage. In fact, the initialization of temperature sensors, amplifiers, analog-to-digital converters, and other circuits does not take as long as $60 \mathrm{~ms}$. The reason for this design is that the output frequency of the PLL needs to be stable for data transmission, and the stability of the PLL in this design takes about $180 \mathrm{~ms}$ (about 300 clock cycles). Figure 8 shows a three-dimensional histogram of the line sensor circuit output stabilization time. In order to make full use of the stabilization time of the PLL, the circuit initialization time of about $60 \mathrm{~ms}$ is artificially set in this design, which will make the previous circuit more stable and increase the robustness of the circuit.

From the simulation results of the steady-state temperature rise of the PVT electric heating field, it can be seen that the output of the temperature sensor is slightly different under different process angles, and the maximum difference is $4.3^{\circ} \mathrm{C}$. From the comprehensive system requirements, the simulation results of the steady-state temperature rise of the electric heating field of the above circuit are acceptable. It can be seen that the output of the temperature sensor rises linearly in the temperature measurement range of $-20^{\circ} \mathrm{C} \sim 120^{\circ} \mathrm{C}$. However, in the design of analog circuits, it is not comprehensive to judge that the circuit is working normally based on the above results, because there will be many process errors in the manufacturing process of integrated circuits, and it is precisely because of this that the process manufacturers are providing the steady-state temperature of the electric heating field. The layered model facilitates the reverse order verification after the experimental function is developed, that is, the design and verification from the bottom module to the high-level module is clear and the purpose is clear. At the same time, it improves the development efficiency and reduces the workload. When the simulation model is upgraded, multiple process angle models are provided for designers to use.

Figure 9 shows the line graph of the circuit's temperature rise conversion efficiency for different input voltages. The main performance indicators of AC-DC circuits include input voltage range, output power, output voltage ripple, power supply regulation, load regulation, and efficiency: (1) when the input voltage is between $5 \mathrm{~V}$ and $10 \mathrm{~V}$, the circuit can work normally, and the chip will not be broken down by voltage. (2) The output voltage is adjustable, the maximum output power is $5 \mathrm{~W}$, and the typical application is $5 \mathrm{~V}-1 \mathrm{~A}$ output. (3) The size of the voltage ripple reflects the stability of the output voltage. The AC-DC circuit designed in this article requires that the output voltage ripple does not exceed 3\%. (4) The linear adjustment rate represents the ability of the output to remain unchanged when the input voltage changes within the allowable input range and is equal to the ratio of the change ratio of the output voltage to the amount of change of the input voltage. The AC-DC circuit designed in this article requires a linear adjustment rate not exceeding 3\%/V. (5) The load regulation rate indicates the ability of the output voltage to remain unchanged when the load of the circuit changes and is equal to the ratio of the change ratio of the output voltage to the change of the load current. The AC-DC circuit designed in this paper requires the load regulation rate not to exceed 15\%/A. (6) The AC-DC circuit designed in this article requires conversion efficiency not less than $85 \%$. 


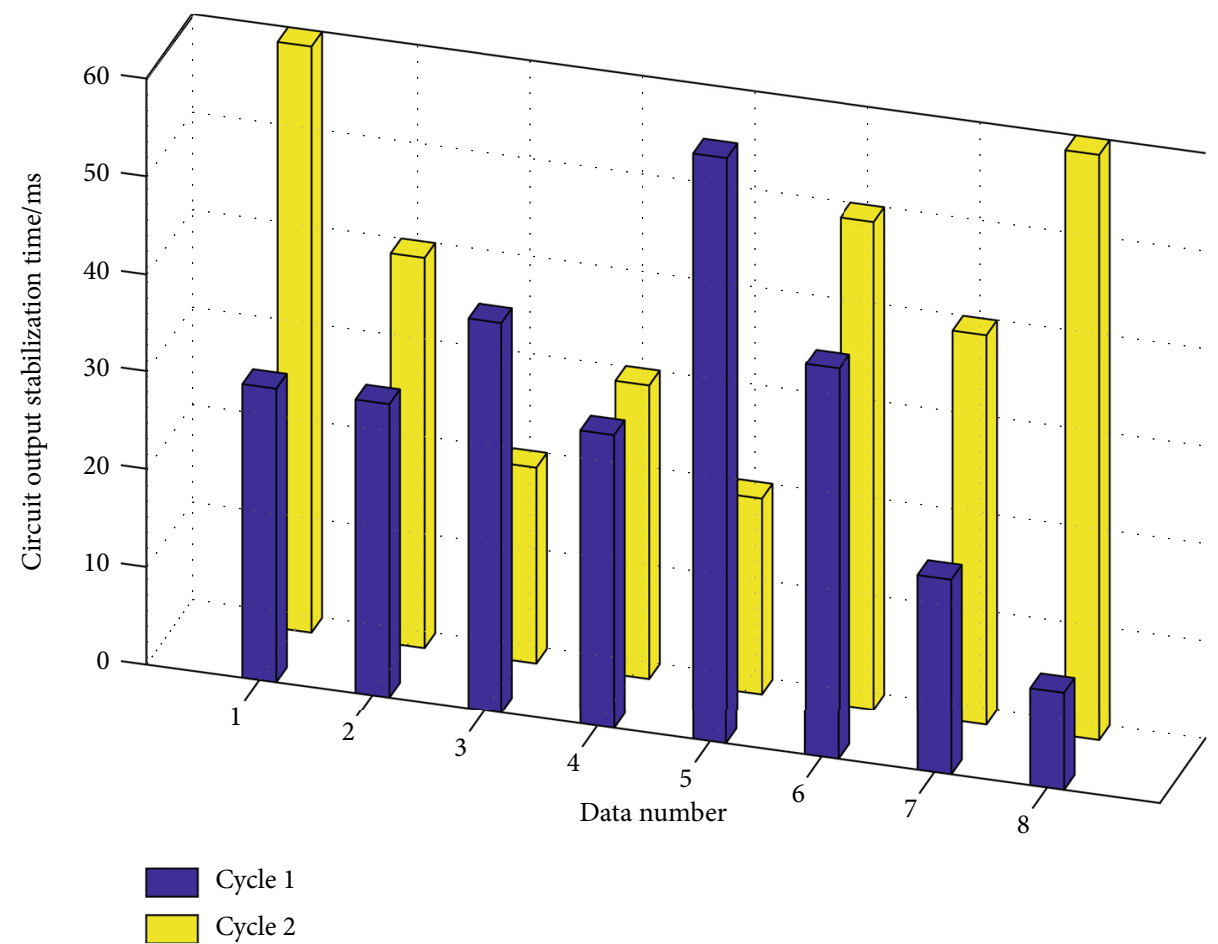

FIgURE 8: The three-dimensional histogram of the output stabilization time of the wireless sensor circuit.

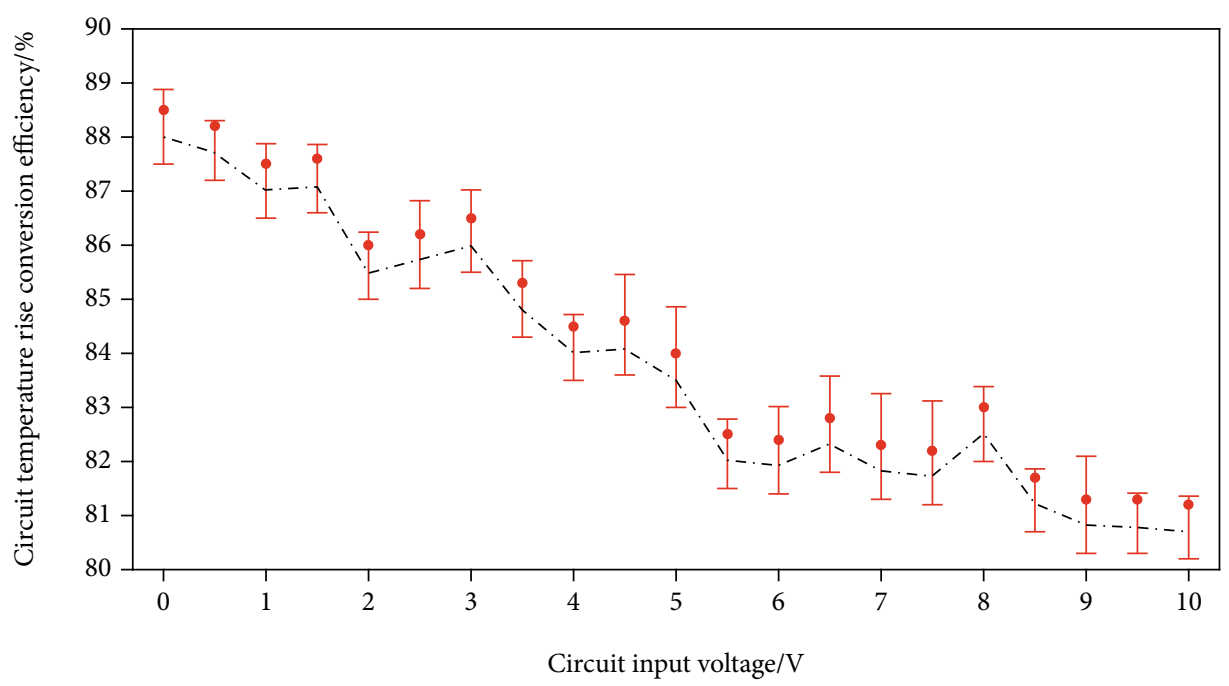

FIGURE 9: Line graph of circuit temperature rise conversion efficiency for different input voltages.

\section{Conclusion}

Based on the wireless sensor circuit technology, a steadystate temperature rise circuit system with high efficiency and high stability is designed in this paper. Through the conversion of the steady-state temperature rise modulation mode of the PWM/PFM electric heating field under different load conditions, the system can operate at full load and achieve high conversion efficiency within the range. The chip adopts the double-loop current control mode, which can feedback the changes of the system and make corresponding response faster, and it has achieved the purpose of improving the dynamic responsiveness and stability of the system. Most of the products currently studied at home and abroad only focus on the efficiency within a certain load range, while the efficiency under heavy load and light load drops rapidly. The conversion efficiency is about $90 \%$ in the full load range, and high stability is achieved to meet the functions and performance indicators required by the circuit design. This paper designs a wireless sensor network system and tests the communication performance of the designed module, which provides a theoretical basis for the application based on RSSI and other parameters. In this paper, through the simulation of the functional structure of 
the virtual digital experiment box, typical chips, and real scenes, a vivid, interactive, and efficient experimental mode is opened. Then, through the radio frequency part of the steady-state temperature rise simulation system of the ADS electric heating field, it can be known that the modified circuit can have better return loss after the steady-state temperature rise simulation of the electric heating field, and impedance matching is better than the original circuit design.

\section{Data Availability}

The data used to support the findings of this study are available from the corresponding author upon request.

\section{Conflicts of Interest}

The authors declare that they have no known competing financial interests or personal relationships that could have appeared to influence the work reported in this paper.

\section{References}

[1] C. Shi, T. Costa, J. Elloian, Y. Zhang, and K. L. Shepard, "A $0.065-\mathrm{mm}^{3}$ monolithically-integrated ultrasonic wireless sensing mote for real-time physiological temperature monitoring," IEEE Transactions on Biomedical Circuits and Systems, vol. 14, no. 3, pp. 412-424, 2020.

[2] S. M. S. Sadati, K. Cetin, H. Ceylan, A. Sassani, and S. Kim, "Energy and thermal performance evaluation of an automated snow and ice removal system at airports using numerical modeling and field measurements," Sustainable Cities and Society, vol. 43, pp. 238-250, 2018.

[3] C. Liang, G. Yang, F. Yuan et al., "Modeling and analysis of thermal characteristics of magnetic coupler for wireless electric vehicle charging system," IEEE Access, vol. 8, pp. 173177173185, 2020.

[4] Z. Xu, J. Yao, Z. Wang et al., "Development of a portable electrical impedance tomography system for biomedical applications," IEEE Sensors Journal, vol. 18, no. 19, pp. 8117-8124, 2018.

[5] H. Wang, A. Jasim, and X. Chen, "Energy harvesting technologies in roadway and bridge for different applications-a comprehensive review," Applied Energy, vol. 212, pp. 1083-1094, 2018.

[6] C. H. Guzmán, J. L. Carrera, H. A. Durán et al., "Implementation of virtual sensors for monitoring temperature in greenhouses using CFD and control," Sensors, vol. 19, no. 1, p. 60, 2019.

[7] K. H. Han and J. Zhang, "Energy-saving building system integration with a smart and low-cost sensing/control network for sustainable and healthy living environments: demonstration case study," Energy and Buildings, vol. 214, p. 109861, 2020.

[8] M. Kazim, A. H. Khawaja, U. Zabit, and Q. Huang, "Fault detection and localization for overhead $11-\mathrm{kV}$ distribution lines with magnetic measurements," IEEE Transactions on Instrumentation and Measurement, vol. 69, no. 5, pp. 20282038, 2020.

[9] X. Ren, C. Li, X. Ma et al., "Design of multi-information fusion based intelligent electrical fire detection system for green buildings," Sustainability, vol. 13, no. 6, p. 3405, 2021.
[10] K. Yazawa, Y. Feng, and N. Lu, "Conformal heat energy harvester on Steam4 pipelines for powering IoT sensors," Energy Conversion and Management, vol. 244, p. 114487, 2021.

[11] M. Shen and Q. Gao, "A review on battery management system from the modeling efforts to its multiapplication and integration," International Journal of Energy Research, vol. 43, no. 10, pp. 5042-5075, 2019.

[12] K. Gao, L. Lyu, H. Huang, C. Fu, F. Chen, and L. Jin, "Insulation defect detection of electrical equipment based on infrared and ultraviolet photoelectric sensing technology," in IECON 2019-45th Annual Conference of the IEEE Industrial Electronics Society, vol. 1, pp. 2184-2189, Lisbon, Portugal, 2019.

[13] A. F. Bendary, A. Y. Abdelaziz, M. M. Ismail, K. Mahmoud, M. Lehtonen, and M. M. F. Darwish, "Proposed ANFIS based approach for fault tracking, detection, clearing and rearrangement for photovoltaic system," Sensors, vol. 21, no. 7, p. 2269, 2021.

[14] S. Gomathi, T. Venkatesan, and D. S. Vidhya, "Design and implementation of fault current limiters in distribution system using Internet of Things," Wireless Personal Communications, vol. 102, no. 4, pp. 2643-2666, 2018.

[15] Y. Wu, L. Qi, H. Zhang, E. M. Musiu, Z. Yang, and P. Wang, "Design of UAV downwash airflow field detection system based on strain effect principle," Sensors, vol. 19, no. 11, p. 2630, 2019.

[16] B. Zaghari, A. S. Weddell, K. Esmaeili et al., "High-temperature self-powered sensing system for a smart bearing in an aircraft jet engine," IEEE Transactions on Instrumentation and Measurement, vol. 69, no. 9, pp. 6165-6174, 2020.

[17] Y. Ji, Q. Tan, H. Wang, W. Lv, H. Dong, and J. Xiong, “A novel surface LC wireless passive temperature sensor applied in ultra-high temperature measurement," IEEE Sensors Journal, vol. 19, no. 1, pp. 105-112, 2019.

[18] A. Mazumdar, Y. Chen, B. G. van Bloemen Waanders, C. F. Brooks, M. A. Kuehl, and M. B. Nemer, "Wireless temperature sensing using permanent magnets for nonlinear feedback control of exothermic polymers," IEEE Sensors Journal, vol. 18, no. 19, pp. 7970-7979, 2018.

[19] C. M. Boutry, L. Beker, Y. Kaizawa et al., "Biodegradable and flexible arterial-pulse sensor for the wireless monitoring of blood flow," Nature Biomedical Engineering, vol. 3, no. 1, pp. 47-57, 2019.

[20] Y. Liu, W. Zhao, G. Liu et al., "Self-powered artificial joint wear debris sensor based on triboelectric nanogenerator," Nano Energy, vol. 85, p. 105967, 2021.

[21] J. H. Li, X. Liao, and C. Chu, "Electromagnetic-thermal-electric analysis of indirectly-heated RF MEMS power sensors with different terminal resistor dimensions," IEEE Sensors Journal, vol. 21, no. 4, pp. 4342-4349, 2021.

[22] Z. Yang, S. Zarabi, E. Fernandes et al., "A simple wireless sensor node system for electricity monitoring applications: design, integration, and testing with different piezoelectric energy harvesters," Sensors, vol. 18, no. 11, p. 3733, 2018. 